\title{
基于 PLC 的煤气报警器数据通讯的应用研究
}

\section{Application Research on data communication of gas alarm based on PLC}

\author{
马连超 綦建钢 \\ Lianchao Ma Jiangang Qi \\ 日照钢铁控股集团有限公司 山东 日照 276806 \\ Rizhao Iron and steel Holding Group Co Ltd Rizhao Shandong 276806
}

摘 要: 为了有效监控公司煤气区域煤气的扩散和泄露, 对固定式煤气报警器数据采集集中监控, 建立在线检测系统。本文 旨在通过 Profinet-Modbus 网关, 实现基于 PLC 的煤气报警器数据通讯的应用研究。应用结果表明系统通讯数据稳定, 使用效 果更加突出。

\begin{abstract}
In order to effectively monitor the diffusion and leakage of gas in the company's gas area, the data collection and centralized monitoring of the fixed gas alarm are carried out, and the online detection system is established. The purpose of this paper is to realize the application research of gas alarm data communication based on PLC through PROFINET Modbus communication gateway. The application results show that the communication data of the system is stable and the application effect is more prominent.
\end{abstract}

关键词: 煤气报警器; PLC; 数据通讯; 应用研究; Profinet-Modbus 通讯网关

Keywords : gas alarm; PLC; data communication; application research; PROFINET Modbus communication gateway

DOI : $10.36012 /$ etr.v2i12.3058

\section{1.引言}

固定式煤气报警器作为公司煤气区域有效监测工具,对 作业人员进入煤气区域提供安全保障。建立在线检测系统可 及时判断泄露覆盖区域, 能够快速发现泄漏点, 便于及时采 取有效的防控和治理措施, 启动相应的应急处置预案。

\section{2. 现状}

目前公司煤气报警器监控系统主要采用以下两种使用 方式:一种煤气报警系统是采用固定式煤气报警器就地显示 报警, 当煤气值超过报警限值语音提示, 此功能形式单一, 对 于煤气泄露频发点无法提前预知并及时防控; 另一种煤气报 警系统是固定式煤气报警器数据采集至集控箱内进行集中 监控, 轮询显示各数据, 且无法查看历史趋势, 如果扩展功能 需要配置一台服务器及系统开发软件进行数据存储, 不便于 系统维护且设备费用消耗较高。

\section{3.系统设计方案}

本系统利用公司现有资源设备, 加热炉区主控 PLC, 应 用 Profinet-Modbus 网关实现与现场固定式煤气报警器实时 数据。其中 Profinet-Modbus 网关作为主站, 多功能报警控制 器作为从站。

\section{1 硬件及结构原理图}

\section{1 .1 硬件}

1)PLC (西门子 S1500):CPU 型号为 6ES7 516-3AN01$0 \mathrm{AB} 0$ 。该系列 $\mathrm{CPU}$ 的硬件设计紧凑,组件和模块高度集成、 通用性强, 通讯速率快的特点。

2)Profinet-Modbus 网关: 网关型号为 WP-PNMD485K20。内置两个 RJ45 以太网接口, 支持 100Base-TX,MDI/ MDIX 自检测, 集成以太网交换机, 可以组成链式网络, 支持 环网究余。

3)多功能报警控制器: 一款循环显示总线、分线控制器。 具有高稳定、高精准和高智能化的特点, 外接控制端口丰富, 可以自由选择挂接传感器的种类, 并通过简单设置即可进行 现场检测与报警。系统配置 32 路 4-20mA 模拟探测器输入, 同时还集成 RS485 通信接口, 便于用户连接工控软件实时监 控设备状态。

4)固定式煤气报警器: 一款自然扩散, 连续在线监测的 气体探测器, 它将检测到的气体浓度或就地显示, 或上传至 在线监测系统进行检测、指示、报警并对报警进行记录和打 印, 以便随时观察和打印留作档案资料。具有隔离最高的防 爆等级、带声光报警功能、可红外遥控校准和越限报警等特 点。

3.1 .2 结构原理

【作者简介】马连超(1987), 工程师,学士, 研究方向: 为自动化技术应用。 
结构原理如图(一)所示, 现场各区域固定式煤气报警器 通过双绞线汇聚至集控箱内, 集控箱集成 RS485 通讯接口通 过 Profinet-Modbus 网关与 PLC 进行数据通讯, 利用人机界 面(HMI) 实时显示。

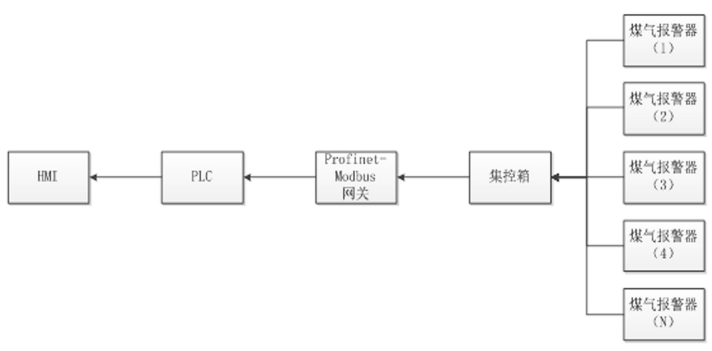

图 1

\section{2 软件组态配置}

系统软件采用 TIA(博图)V15.1 版本, WINCC V7.4sp1。

3.2.1 PLC 网络配置:

打开 TIA（博图）软件，在项目视图的选项中安装 Profinet-Modbus 网关 GSD 文件 “GSDML-V2.25-WINGPNMDMM_Vx.xx-xxxxxx.xml”。在设备组态的网络视图窗口 中添加 Profinet-Modbus 网关 GSD 文件至 PLC 子网 PN/IE_1 并分配设备名称, Profinet-Modbus 网关 IP 地址设置为 192.168.51.3,PLC 网络 IP 地址为 192.168.51.2。

3.2 .2 主站及从站通讯接口参数配置:

1 )Modbus Baudrate(波特率): 主站和从站设备选择相一 致, 默认选择为 $9600 \mathrm{bps}$ 。

2) Modbus Parity（校验): 选择 8 位无校验 1 停止位( 8 DataLen, 1 StopBit, No Parity)。

3 )Modbus Reply Time(等待应答时间): 设置为 $2 \mathrm{~S}$, 现场 共计 32 个从站数量多, 避免从站应答时间超时。

4 )Modbus Idle Time（总线空闲时间）：选择默认设置 $50 \mathrm{~ms}$ ，即主站接受到从站应答报文后等待 $50 \mathrm{~ms}$ 再发送下一 条报文。

5 )Modbus Slave Monitor (从站状态检测): 选择有从站状 态检测 32 位(Monitor 32 Bits)。

6 )主站网关通讯控制字及状态字默认为 10 。

\subsubsection{Modbus 报文设置:}

添加 32 条从站报文读取（4XXXX-03H Read 2 Words） 和 1 条从站报文写入 $(4 X X X X-10 \mathrm{H}$ Write 2 Words $)$ 。Modbus No (从站地址)依次从 1 至 32 , Start Address(起始地址)设置 为 7012 (即 7012 对应煤气浓度)。 3.2.4 PLC 控制程序编译:
如图 (二) 所示, 建立功能块 $(\mathrm{FC})$ 及数据块 (DB), 编写 PLC 控制程序，根据网关通讯控制字中第 0 位定义启动工 作，程序内保持 Q10.0 置 1 状态。因从站返回数据为 32 位 IEEE 浮点型，程序内读取起始地址参照从站报文通道进行 访问, 采集数据顺序对应从站地址配置顺序。

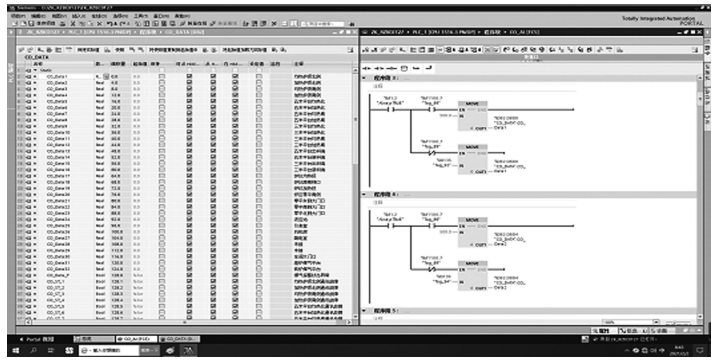

图 2

3.2.5 WINCC 画面编译:

如图(三)所示, WINCC 内建立数据变量, 地址同 PLC 数 据块地址, 数据类型相同。建立过程监控画面, 根据现场各煤 气报警器安装位置添加至画面中进行注释说明。当某一固定 式煤气报警器浓度超过 $25 \mathrm{ppm}$ 时, 画面启动报警显示功能, 另增加历史趋势供操作人员进行查看。

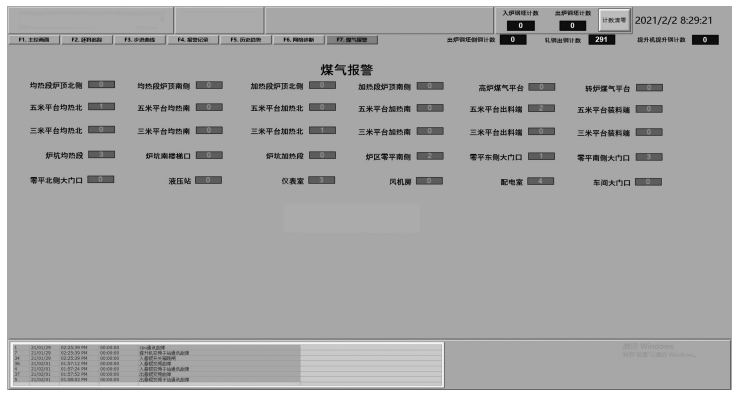

图 3

\section{4. 结语}

通过对煤气报警器数据远程采集监控, 网络通讯数据更 加稳定, HMI 画面集中显示更加直观, 系统可扩展能力更强。 利用煤气浓度实时数据的观测, 更利于操作人员对现场作业 环境煤气浓度有效辨识, 便于及时采取有效的防控和治理措 施, 为公司安全生产提供有利保障。

\section{参考文献}

[1] 郭翠娟,李中原, 荣锋.PROFINET-Modbus 通信网关的设计[J]. 仪表技术与传感器,2020(09):121-126.

[2] 封帆,李玉才, 蔡海峰.焦化厂煤气监测、报警系统改造[J].自动化 应用,2018(05):29-30.

[3] 崔坚.SIM ATIC S7-1500 与 TIA 博途软件使用指南 [M].北 京: 机械工业出版社,2016.177-260. 